(Physiologisches Laboratorium in Bonn.)

\title{
Ueber die elementare Zusammensetzung des Ochsenfleisches.
}

Von

P. Argutingky.

Es ist eine merkwürdige Thatsache, dass unsere Kenntniss einer der wichtigsten Fragen der Ernährungsphysiologie, die der elementaren Zusammensetzung des Fleisches, noch immer eine sehr unvollständige ist. Wenn wir von den älteren Angaben absehen, so ist diese Frage in den letzten Jahren nur von wenigen Forschern und man kann sagen, nicht eingehend genug behandelt worden.

Die bis jetzt vorhandenen Elementaranalysen des Fleisches sind sehr gering an der Zahl und weichen beträchtlich von einander ab. Wir wissen nicht, ob die verschiedenen Muskeln desselben Thieres, oder die gleichen Muskeln verschiedener zu derselben Species gehörender Thiere eine übereinstimmende Elementarzusammensetzung haben, und ob etwa Ernährungsweise resp. Hungerzustand, die Ruhe und die Arbeit die Zusammensetzung des Muskels beeinflussen. Vor Allem aber fehlt uns eine Methode, die Muskelsubstanz möglichst ohne Aenderung ihrer Zusammensetzung zur Elementaranalyse vorzubereiten.

Die nachfolgende Untersuchung bildet einen Theil einer Versuchsreihe, die zur Beantwortung der oben angefuihrten Fragen unternommen wurde, und sucht zwei Aufgaben zu erledigen: 1) eine Methode der Muskeluntersuchung auszuarbeiten und 2) die Zusammensetzung des Fleisches beim Ochsen resp. Kuh festzustellen.

\section{Methode der Muskeluntersuchung.}

Es handelt sich bei unserer Aufgabe um die Ermittlung des Gehaltes der Fleischsubstanz an N, C, H, O und um die Bestim- 
mung der Fleischasche, wobei es selbstrerständlich erforderlich ist, stets mit möglichst unverändertem Fleische zu arbeiten. Namentlich kommt es darauf an, dass die Ergebnisse der organischen Elementaranalyse die Verhältnisse der ganz frischen Fleischsubstanz auch nach stattgehabter vorbereitender Bearbeitung des Fleisches genat wiedergeben.

Bei der ersten Ueberlegung könnte es scheinen, als ob es am vortheilbaftesten wäre, die analytische Untersuchung unmittelbar an frischem Fleische vorzunebmen. Indessen bei genauer Pritung ist diese Annahme durchaus zu verwerfen. Denn die Bestimmung des Kohlenstoffgehaltes direct im frischen Fleische muss immer fehlerhaft sein und zwar aus folgenden Gründen:

1. Da das Fett einen beinahe 7 fach höheren Kohlenstoffgehalt besitzt als das frische Fleisch selbst, so beeinflusst die nicht zu vermeidende geringe Fettbeimengung sehr bedeutend die analytischen Resultate und dieses um so mebr, da aus praktischen Gründen nur kleine Mengen, höchstens 1,0-1,5 Gramm frischen Fleisches zur Analyse verwendet werden können. Dass gerade in diesen so geringen Quantitäten Fleisches jedesmal die Durchschnittsmenge des dem Fleische beigemengten Fettes enthalten sein sollte, schien sebr unwahrscheinlich und ist auch, wie die Analysen mir ergeben haben, thatsächlich nicht der Fall.

2. Ausserdem aber ist das zur Ausfiihrung der Analyse nothwendige feine Zerschneiden so kleiner abgewogener Quantitäten frischen Fleisches ohne empfindlichen Verlust an Substanz und dadurch bedingte Ungenauigkeiten der analytischen Ergebnisse kaum möglich.

Ferner ist eine genaue Bestimmung des Wasserstoffgehaltes der Fleischsubstanz durch die elementare Analyse frischen Fleisches und nachträgliche Feststellung seines Wassergehaltes ebenfalls kaum zu erreichen.

Sogar die Bestimmung des N-Gehaltes im Fleische nach Kjeldahl-Wilfarth (welche Methode den Vortheil darbietet, die Verwendung grösserer Fleischquantitäten zur Analyse zu gestatten) giebt, angewandt beei frischem Fleische, keine so durchaus ïbereinstimmende Zahlen, als nach vorhergegangener Trocknung und Entfettung.

Nachdem es sich herausgestellt hatte, dass nothwendig mit dem getrockneten Fleische gearbeitet werden muss, habe ich verschie- 
dene Wege zur Trocknung des Fleisches eingeschlagen. Dem Trocknen aber, nachdem das Fleisch von sichtbaren Beimengungen von Fett und Sehnen nach Möglichkeit befreit war, musste natürlich jedesmal die Zerkleinerung vorhergehen.

Selbstverständlich ist das feine Zerschneiden des Fleisches mit der Scheere und dem Messer bei grösserer Fleischmenge zu mühsam und zu langwierig und lässt das Ziel nur sehr unvollkommen erreichen.

Wie ich mich durch vielfache Analysen iberzeugt habe, eignet sich für unsere Zwecke auch die Zerkleinerung des Fleisches mit den sogenannten Hackmaschinen durehaus nicht. Man mag noch so vorsichtig mit der Maschine verfahren, sie schneidet nicht nur, sondern presst zugleich und quetscht, wodurch dem zerkleinerten Fleische stets der ausgepresste Fleischsaft und zwar ungleichmässig vertheilt beigemengt ist, während in der Hackmaschine ausser Sehnen noch ausgepresstes Fleisch zurückbleibt.

Allen Anforderungen für unsere Zwecke genügt das sorgfältige Zerkleinern des Fleisches mit einem scharfen Hackmesser auf einem glatten harten Holzbrett (Buchenholz) ${ }^{1}$ ), was sehr rasch vor sich geht (in etwa 5 Minuten) und einen Brei von ausserordentlicher Gleichmässigkeit und Feinheit liefert. Der feingeschnittene Fleichbrei verändert sich sehr schnell - säuert, verfärbt sich und fault - ausserdem sickert schon nach wenigen Stunden Fleischsaft aus den oberen in die unteren Breischichten und der Brei wird ungleich. Aus diesen Gründen ist es nothwendig, dass der Zerkleinerung sofort die Trocknung folge und diese nach einer Methode geschehe, die ein rasehes Trocknen des Fleischbreis ermöglicht.

1. Das Trocknen und Entfetten des Fleisches.

Das Trocknen bei Zimmertemperatur, sowohl frei in der Luft als im Exsiccator über Schwefelsäure erwies sich, wie es zu erwarten war, als ganz unbrauchbar. Das Troeknen geht dabei so langsam, dass der Fleischbrei in allen meinen hierhergehörigen Versuchen stellenweise verfanlte und mit einer dem Auge sichtbaren Pilzvegetation

1) Das Brett wird jedesmal vor Gebrauch glatt gehobelt. 
sich bedeckte, auch einen widerlichen, säuerlichen Geruch entwikkelte. Erst nach mehreren Tagen trocknete er za einem festen, dunkelverfärbten Kuchen, der so hart und dabei so zähe war, dass er sich auf keine Weise pulvern liess.

Hierauf versuchte ich das Trocknen bei höherer Temperatur. Ich habe den Fleischbrei in ganz dunner Schicht auf einer glasirten Blechpfanne ausgebreitet und die letztere durch lebhaften Dampfstrom erhitzt, der in einem von der Pfanne bedeckten Wasserbehälter entwickelt wurde. Das Fleisch veränderte sehr schnell seine Farbe, (sah wie abgebrïht ans), dampfte längere Zeit und verbreitete einen intensiven Geruch. Ein in den dampfenden Fleischbrei eingelegter feiner Thermometer zeigte 90 bis $92{ }^{\circ} \mathrm{C}$. In einigen Stunden war der Brei getrocknet, batte nun eine dunkelbraune Färbung, zeigte sich sehr brïchig, spröde und liess sich gut zu einem groben, braunen Pulver zerreiben. Da ich nun annehmen musste, dass die mehrstündige Einwirkung so hoher Temperaturen auf das feuchte Fleisch unmöglich ohne Beeinflussung seiner Zusammensetzung bleiben konnte, so wählte ich darauf weniger hohe Temperaturen und bediente mich zu diesem Zwecke des Trocknens in einem grossen geräumigen Lufttrockenschrank, regulirt anf etwa $50-60^{\circ} \mathrm{C}$. Aber auch dies Verfahren, da es bedeutend mehr Zeit verlangt (bis einen Tag und darẗber), erweist sich als unbrauchbar. (Es liefert ebenfalls einen dunkelbraunen, spröden, brïchigen, leicht zu einem groben Pulver zerreibbaren Trockenriickstand.)

Ganz dasselbe Verhalten wie der Fleischbrei, zeigte auch das in feinen dünnen Scheiben zerschnittene Fleisch (Stohmann und Langbein). Bei Zimmertemperatur ging das Trocknen zu langsam, bei höherer dagegen hatte man mit dem oben erwähnten Uebelstande zu rechnen.

Von der Ueberlegung ausgehend, dass alle die erwähnten Trocknungsmethoden aus den oben entwickelten Gründen unzulänglich sind, habe ich nach einer anderen besseren gesucht, welche bei Vermeidung höherer Temperatur ein s e hnelles und gleichmässiges 'Trocknen ermöglicht, und bin dabei zu folgendem, diese Bedingungen erfüllendem Verfahren gekommen, welches ich nicht genug empfehlen kann, da es ebenso aufMuskeln, wie auf verschiedene andere Gewebe Anwendung findet und viel schneller zum Ziele fiuhrt, als das Trocknen bei hoher Temperatur. 
Das Verfahren besteht in der Hauptsache in Folgendem:

Aus einem Drahtnetzgewebe ron Nickel, wie es im Handel leicht zu haben ist, werden mehrere (etwa 4) runde Scheiben von $15-16 \mathrm{~cm}$ Durchmesser mit der Scheere geschnitten und der Rand einer jeden Scheibe rund herum mit den Fingern nach einer Seite umgebogen, so dass die Scheibe zu einem Teller wird. Ausserdem werden von demselben Drahtnetzgewebe $2 \mathrm{~cm}$ breite und $40-50 \mathrm{~cm}$ lange Streifen geschnitten und jeder einzelne Streifen zu einem geschlossenen Reifen (Ring) von etwa 10-12 cm Durchmesser gebogen.

Auf jeder Drahtnetzscheibe (nach vorhergegangenem vorsichtigem Erbitzen derselben zum Zwecke der Reinigung) wird etwa 30-35 Gramm Fleischbrei mittelst zweier Scalpelle möglichst flach und gleichmässig ausgebreitet. Nachdem etwa 4 solcher Drahtnetzscheiben auf beschriebene Weise mit Fleischbrei beschickt sind, werden dieselben tellersatzartig auf einander gebaut, indem man auf jede Scheibe einen Streifenring legt, der der nächst oberen Scheibe als Unterlage dient. Diese so hergestellte Etagère (Drahtnetzgestell) wird auf eine mit Drabtnetz bedeckte Krystallisirschale gestellt, die eine etwa $2 \mathrm{~cm}$ hobe Schicht reinster concentrirter Schwefelsäure enthält; das Ganze - Schale und Gestell - wird dann unter eine Glocke gebracht. Die Glocke, von eirca $18-20 \mathrm{~cm}$ Durchmesser und $30-35 \mathrm{~cm}$ Höhe, mit nicht allzu dünnen Wänden versehen und auf einer starken (dicken) Glasplatte aufgeschliffen, besitzt nur einen (seitlichen) Tubulus, der mittelst einer mit Hahn versehenen Seitenröhre und einem dicken unnachgiebigen Gummirohr mit einer Quecksilberpumpe in Verbindung gebracht, vollständig evacuirt and luftdicht abgeschlossen werden kann. Die vorher erwähnte Krystallisirschale mit Schwefelsäure ist ziemlich hoch (10$12 \mathrm{~cm}$ ) und bleibt im Durchmesser nur wenig hinter dem inneren Durchmesser der Glocke.

Direct nach dem Einbringen des mit Fleisehbrei beschickten Drahtnetzgestelles (der Etagère) in die Glocke, wird diese vollständig evacuirt. Das danert etwa $1 / 2$ bis $3 / 4$ Stunden, natürlich je nach dem (Grössen-) Verhältniss der Pumpe zum Kubikinhalt der Glocke. Sobald der Druck in der Glocke bedentend gefallen ist, sicht man bei jedem Oeffnen des grossen Hahnes der Pumpe eine leichte Dampfwolke vom Fleischbrei aus aufsteigen, anfangs sehr deutlich sichtbar, nach weiterem Wasserverlust des Fleisch- 
breis immer undeutlicher werdend. Ist dann der Druck beinahe auf Null gesunken, so sieht man bei fortgesetztem Pumpen das Fleisch in der Glocke schnell (in wenigen Minuten) sein Aussehen ändern. Es verliert vollständig seinen Glanz, wird blässer nnd bekommt durchgehends eine poröse Beschaffenheit (ähnlich wie eine aufgeblasene Lunge), indem es, wie man sich leicht überzeugen kann, in seiner ganzen Dicke von feinsten (eben sicbtbaren) Hohlräumen durchsetzt wird, und siebt nun ganz trocken aus. Nimmt man jetzt das Fleisch, nachdem die Evacuation vollständig beendet ist, ans der Glocke heraus, so kann es sogleich verpulvert werden. Ich habe aber meist vorgezogen, dasselbe noch 24 Stunden in der Glocke stehen zu lassen, habe es dann mit einem Scalpellstiel von den Drahtnetzscheiben abgeschabt (es geht sebr leicht) und zur Verpulverung und Entfettung verwandt ${ }^{1}$ ).

Von grösster praktischer Wichtigkeit ist es, dass so getrocknetes und in einer evacuirten und luftdicht abgeschlossenen Glocke befindliches Fleisch unbegrenzt lange in derselben aufbewabrt werden kann, ohne irgend eine Veränderung seines Aussebens und seiner Zusammensetzung zu erleiden. Deshalb kann auch die nachfolgende Entfettung und weitere Behandlung des getrockneten Fleisches beliebig lange aufgeschoben werden.

Nur wenige Worte ïber die Entfettung des Fleisches.

Ich habe anfangs die Entfettung des Fleisehes nach zwei Methoden vorgenommen. Ich habe sowohl mit warmem Aether im Soxhlet'sehen Apparat extrahirt, als in anderen Fällen das Fleisch wochenlang im kalten Aether (Zimmertemperatur) bei täglichem Umrühren und mehrmaligem Wechsel des Aethers stehen lassen. Mag diese letztere Methode, wenn man mit kleinen Quantitäten Fleisehes (wenige Gramm) arbeitet, gute Resultate erzielen, bei grösseren Mengen Fleischpulvers $(20-30 \mathrm{gr})$ führte sie mich nie zum Ziel. Selbst nach wochenlangem Stehenlassen waren nicht zu vernachlässigende Reste des Fettes vorhanden, wie es die nachträgliche Extraction nach Soxhlet bewiesen hat. Daher bin ich im weiteren Verlaufe der Arbeit stets nach Soxhlet verfahren.

Auch diese Extraction nach Soxhlet muss, um ibren Zweck zu erfüllen, lange Zeit fortgesetzt werden. Ich war manchmal ge-

1) Statt des Drahtnetztellers kann man im Nothfall ebenso gut einen Glasteller resp. ein grosses Uhrglas benutzen. 
nöthigt, eine Woche lang dasselbe Fleisch zu extrahiren. Zwar erhält man bereits am ersten Tage die Hauptmenge des Fettes, und der Ertrag der Extraction am zweiten Tage beträgt kaum $1 / 20-1 / 30$ des ersten, doch bleibt auch am dritten und vierten Tage nach Abdunstung des Aethers immer etwas Fett im Rückstand, welches erst später, etwa am fïnften, sechsten Tage nur wenige Milligramme beträgt ${ }^{1}$ ). Auf diesem Punkt angelangt, habe ich in der Regel die Extraction als beendet angesehen, da es mir auch nach weiter fortgesetzter Extraction nie gelang, den Aether frei von jeglichem Ruickstand zu erhalten.

Man könnte den Einwand machen, dass die an jedem der letzten Extractionstage erhaltenen wenigen Milligramme Aetherextracts, welche im Verbältniss zur verwendeten grossen Fleischmenge (10-20 gr und mehr) vernachlässigt werden konnten, doch, wenn dieselben viele Tage beobachtet wiurden, eine wohl zu beriicksichtigende Summe bildeten. Ohne die Berechtigung dieses Einwandes bestreiten zu wollen, muss ich doch sagen, dass eine eine Woche dauernde Fettextraction mit schnell eirculirendem warmen Aether im Soxhlet'schen Apparat wohl die Grenze dessen erreicht, was man praktisch verlangen kann.

Ich habe noch hinzuzufügen, dass die tadellose Feinheit des Fleischpulvers eine grosse Rolle bei erfolgreicher Fettextraction spielt. Der getrocknete Fleischbrei, so spröde und brïchig er auch ist, lässt sich, wie erwähnt, doch nur grob verpulvern und fein erst, wenn der grösste Theil seines Fettes entfernt ist. Deshalb muss man stets das Fleisch, nachdem es 1 bis 2 Tage nach $\mathrm{S}$ ox hlet behandelt worden ist, aus dem Apparat herausnehmen und erst nach feinster Verpulverung desselben die Extraction fortsetzen. Ja, zuweilen musste diese Operation zum zweiten Male wiederholt werden.

Nach der Extrahirung des Fettes wird das von Aether durchfeuchtete Fleisch so lange in einem grossen Mörser zerrieben, bis der Aether verdunstet ist, sodann das noch stark nach Aether riechende Pulver sogleich in einen Exsiccator gelegt, der sowohl Schwe-

1) Während in den ersten Tagen der Rückstand eine ölige, fettige Beschaffenheit zeigte, war der spärliche Rückstand der letzten Tage krystallinischer und liess die Annahme zu, dass wir es hier mit einem anderen Körper zu thun hatten, als mit Fett. 
felsäure als Paraffin (zur Absorption des Aethers) enthält und darin etwa einen Tag stehen gelassen, bis kein Aethergeruch am Fleische mehr wabrzunehmen ist.

Das so behandelte Fleischpulver enthält noch, da es in hohem Grade hygroscopisch ist, wechselnde Mengen von Wasser. Bevor es zur Analyse verwendet werden kann, muss es daber, in mebrere Wägegläschen vertheilt, iiber Schwefelsäure in vacuo bis zum Erreichen des constanten Gewichts nachgetrocknet werden. Wenn das Fleischpulver in jedem Wägegläschen nur eine niedrige Schicht bildet und die Glocke absolut luftdicht schliesst, so ist das in circa 1, höchstens $1 \frac{1}{2}$ Wochen geschehen, wobei man nur ungefähr alle drei Tage die Glocke zum Wägen der Gläschen zu lüften hat.

Die hochgradige hygroscopische Eigenschaft des Fleischpulvers macht es durchaus nothwendig, dasselbe auch nach dem Erreichen des constanten Gewichts, soweit es nur geht, in der luftleeren Glocke aufzubewahren, was zugleich auch die mögliche Beeinflussung des Pulvers durch langdauernde Berührung mit dem atmosphärischen Sauerstoff ausschliesst. Nach der Entnahme aus dem Vacuum sind die Wägegläschen nicht allein gut verschlossen zu halten, sondern auch stets im Exsiccator über Schwefelsäure aufzubewahren.

Wenn man sich vielfach überzeugt hat, dass das in unbedeckter Schale frei in der Luft stehende Fleischpulver in wenigen Tagen um 15\% seines Gewichts und mehr durch Wasseraufnahme zunehmen kann, ohne dabei in seinem Aussehen anch die geringste Aenderung darzubieten, so kann man beurtheilen, wie grosse Fehlerquellen für die Analysen damit gegeben sind und wie sorgfältig die hygroscopische Eigenschaft des Fleischpulvers berücksichtigt werden muss. Noch eine andere nnliebsame Eigensehaft des Fleischpulvers verlangt ebenfalls eine sorgfältige Beachtung. Es stäubt nämlich das Fleischpulver wegen seiner ausserordentlichen Feinheit sehr stark, und man muss daher, namentlich beim Luiften der Wägegläschen, um jeden Verlust zu vermeiden, stets vorsichtig verfahren.

\section{Analytische Methoden.}

$\mathrm{Zu}$ sämmtlichen Analysen diente allein und ausschliesslich das bis zum constanten Gewicht in vacuo bei Zimmertemperatur getrocknete Fleischpulver, welches bei jeder Fleischart auf mehrere 
breite, gut schliessende Wägegläschen vertheilt war. Ein jedes Gläschen enthielt etwa 2,0 bis 4,0 Gramm.

Um von dem leicht stäubenden und sehr hygroscopischen Fleischpulver eine genau bestimmte Menge aus dem Wägegläschen in eine enge Röhre (Zinke'sche Röbre, siehe unten), in ein Kölbchen ete, überführen zu können, habe ich folgendes Verfahren eingeschlagen :

In das Wägegläschen mit dem Fleischpulver wurde ein Spatel, das heisst eine längliche Platinblechrinne, eingelegt und das Gläschen sofort wieder gut verschlossen. Da die Länge des Spatels die Breite des Wägegläschens bedentend übertraf, so tauchte er nur mit seinem unteren Theile in das Fleischpulver und kam äberhaupt nur mit diesem Ende mit dem Fleischpulver in Berührung.

Das Gläschen wurde nun zweimal gewogen. Nach der ersten Wägung wurde bei schneller Luiftung des Glasdeckels der Platinspatel rasch mit einer passenden Schieberpincette an seinem oberen Ende gefasst, und die gewünschte Menge des Fleischpulvers in das neben dem Wägegläschen stehende erforderliche Gefäss unter ïblichen Vorsichtsmassregeln übergefübrt, ohne auch nur die geringste Spur zu verlieren, und sogleich der Spatel mit dem daran noch haftenden Fleischpulver in das Wägegläschen zurückgebracht, der Glasdeckel aufgesetzt, und das Wägegläschen abermals gewogen.

Die Differenz ergab das g e n a e Gewicht der zur Analyse entnommenen Fleischprobe.

Es wurden an jeder untersuchten Fleischart Aschen- und Glycogenbestimmungen gemacht, der Stickstoff nach KjeldahlWilfarth ermittelt, und zur Analyse des Kohlenstoffs und des Wasserstoffs Verbrennungen im offenen Rohr unter Sanerstoffstrom ausgeführt.

1. Die Aschenbestimmung geschah folgendermassen: 1,0 $1,5 \mathrm{gr}$. Fleischpulver wurden in einem Platintiegel anhaltend aber nur schwach erhitzt und zwar so, dass das Fleischpulver gar nicht oder nur ganz wenig rauchte. Die Erhitzung habe ich stets iber Nacht fortgesetzt, wobei sorgfältig darauf geachtet wurde, dass der Platintiegel - erst offen, nach einigen Stunden bedeckt - gar nicht in Berührung mit der Flamme des Gasbrenners kam. Am folgenden Tage enthielt der Tiegel stets glanzlose, zusammengebackene Kohle, die nun zur Trennung der löslichen und unlöslichen Aschenbestandtheile mit kochendem Wasser übergossen im Platin- 
tiegel selbst mit einem dicken Glasstab möglichst fein zerrieben und nach Ueberführung auf ein aschefreies Filter sorgfältig mit heissem Wasser ausgewaschen wurde. Das Filtrat - es war fast farblos, wenn die Erhitzung der Kohle lange genug fortgesetzt war - wurde in einer Platin-Schale auf einem Wasserbade zur Troekne abgedampft und nach leichtem, wenige Secunden dauernden Erhitzen iiber der Gasflamme (zur Verbrennung der selır geringen Beimengungen der feinst vertheilten Koble) in einem Exsiceator über Schwefelsäure erkalten gelassen und gewogen. Hierauf wurde das auf dem Filter gebliebene nasse ausgezogene Kohlenpulver nebst Filter in den Platintiegel gebracht, und der Tiegel nach Abdampfen des Wassers (in einem Trockenschrank, oder besser auf einem Wasserbad) bis zur schwachen Rothglut einige Stunden erhitzt. Der hiernach bleibende weisse Riiekstand bildete, wenn vorher die Kohle von löslichen Salzen sorgfältig befreit war, ein amorphes, nicht sehmelzbares Pulver, das, wie uiblich, nach Erkalten des Tiegels im Exsiccator iuber Schwefelsäure, gewogen wurde.

Die löslichen und unlöslichen Bestandtheile gaben zusammen die Asche des getrockneten entfetteten Muskels.

Ich habe bei der Aschenbestimming folgende Punkte besonders beachtet:

Erstens wendete ich vor Entfernung der Chloride und Carbonate (vor dem Ausziehen der Kohle mit Wasser) nur eine möglichst sehwache Erhitzung an.

Zweitens nahm ich das Ausziehen der Kohle mit kochendem Wasser erst nach vollständigem Verkohlen der organischen Substanz vor, um so das Filtrat - die löslichen Salze - vor Beimengung organischer Substanzen zu bewahren, denn sonst ist eine nachträgliche starke Erhitzung des eingedampften Filtrats, zum Zweck der Verbrennung der organischen Substanzen, nothwendig; was gewiss mit Verlust der flüchtigen löslichen Salze verbunden ist.

Drittens erhitzte ich die Kohle zum schwachen Rothglïhen erst nach nachträglicher Entfernung aller löslichen Salze.

2. Die Glycogenbestimmug geschah - stets an in vacuo getrocknetem Fleische - nach Brücke-Külz'scher Methode, nach den von $\mathrm{K} u \mathrm{ilz}$ gegebenen Vorschriften.

3. Ein Punkt, die Stickstoff bestimmung betreffend, die immer nach $K j$ eldahl-Wilfarth ausgefiihrt wurde (dies Archiv, Bd. 46, Seite 33), muss hier genau erörtert werden, da er sonst 
trotz der Zuverlässigkeit der Methode zur Quelle unliebsamer Fehler werden kann.

Es benetzt sich nämlich merkwürdiger Weise das feine getrocknete Fleischpulver nicht mit der Schwefelsäure. Das bedingt, dass im Oxydationskölbchen das Fleischpulver zum grösseren oder kleineren Theile auf der Schwefelsäure schwimmt und bei der Erhitzung der letzteren, da es von ihr nicht benetzt ist, einer trockenen Destillation unterworfen wird. Diese hat selbstverständlich Stickstoffverlust zur Folge. Daher stimmen die Analysen in diesen Fällen nicht iuberein und geben, wit ich mich noch nachträglich überzeugt habe, sehr leicht ein Deficit an Stickstoff. Zur vollständigen Beseitignng dieses Uebelstandes genügt es, das in dem Oxydationskölbchen enthaltene Fleischpulver vor dem Zugiessen der Schwefelsäure mit 2-3 ccm destillirten Wassers zu benetzen, wonach die Schwefelsämre dann das nasse Fleischpulver leicht und vollständig durchdringt.

Die einzelnen Analysen desselben Fleischpulvers nach Kjeldahl-Wilfarth zeigen eine ganz merkwürdige Uebereinstimmung. Die Differenzen, obwohl das trockene Fleisch um $4-41 / 2$ mal reicher an Stickstoff ist als das frische, bewegen sich meist nur in Hundertsteln eines Procents und übersteigen nie $1 \frac{1 / 2}{2}$ Zehntel Procent, sind also viel tibereinstimmender, als die Analysen frischen Fleisches.

Wenn ich auch keine Bestimmungen des Stickstoffs im Fleische zugleich nach der Methode von Dumas gemacht habe, so glaube ich doch annehmen zu dürfen, dass die nach $\mathrm{Kjeldahl-}$ Wilfarth enthaltenen Resultate in der That dem gesammten Stickstoffgehalt des Fleisches entsprechen. Zwar findet die Kjel. dahl-Wilfart h'sche Methode nicht auf alle Stickstoffverbindungen ihre Anwendung, es sind aber die ihr nicht zugänglichen Verbindungen entweder im Fleische gar nicht enthalten oder nur in zu vernachlässigenden minimalsten Spuren.

4. Die Koblenstoff- und Wasserst offbestimmung geschah, wie bereits erwähnt, durch Verbrennung im offenen Rohr im Sauerstoffstrom.

Nach Ausführung einer grossen Zahl von Verbrennungen muss ich sagen, dass diese Methode sicherlich eine bei Weitem häufigere Anwendung zu thierphysiologischen und klinischen Untersuchungen finden kann, als sie bis jetzt gefunden bat, wenn sie 
auch bedeutend complicirter und zeitraubender ist, als die $\mathrm{Kjel}$ $\mathrm{d}$ a hl'sche Stickstoff bestimmung und, um gute Resultate zu liefern, Einhaltung vieler Bedingungen verlangt. Nach einiger Uebung, die rasch zu erreichen ist, liefert sie Resultate, die an Genauigkeit wenig denen der Stickstoffbestimmung nach $\mathrm{KJ}$ eldahl nachstehen.

Wie selbstverständlich, hatte ich durch öfters wiederholte Verbrennungen chemisch reiner Körper mich von Zeit zu Zeit immer wieder überzengt, dass die Verbrennungsresultate nicht allein unter einander übereinstimmtın, sondern auch zuverlässig waren.

\section{Analysen des Fleisches.}

1. Vorbemerkungen.

Die zu nachfolgenden Analysen verwendeten Fleischportionen stammten sämmtlich von in meiner Gegenwart im Bonner Scblachthause geschlachtetem Vieh, auch waren die Thiere, denen ich das Fleisch entnommen hatte, alle auf eine und dieselbe Weise Einschlagen eines Stifts in den Schädel und darauf folgende Eröffnung der Halsgefässe - getötet.

Das in Staniolblatt eingewickelte Fleisch wurde rasch in das nur 10 Minuten entfernte physiologische Laboratorium gebracht, darauf nach Entfernung mit Messer and Scheere von sichtbaren Beimengungen von Fett und Sehnen, fein zerhackt, der Brei in einer luftleeren Glocke getrocknet und auf die oben beschriebene Weise weiter behandelt.

Schlachteten wir im Laboratorium selbst - wir haben einige Tauben und ein paar Hunde geschlachtet, also verhältnissmässig nicht zu grosse Thiere, - so konnten wir das Fleiseh in höchstens einer halben Stunde schon zerhacken und nach weiteren $1 / 2$ bis $3 / 4$ Stunden war der Fleischbrei in der evacuirten Glocke bereits trocken. Hat man aber, wie in unserem Falle, mit grossen Schlachtthieren zu thun, so vergeht, wie selbstverständlich, vom Moment des Schlachtens bis man das Fleisch zerbacken kann, verhältnissmässig nicht wenig Zeit, eben weil die Exenteration, Enthäutung und Zerlegung des grossen Schlachtthiers Zeit in Anspruch nimmt. Immerhin aber war das Fleisch schon nach höchstens 4 Stunden zum Zerhacken bereit und nach weiteren $3 / 4$ Stunden der Fleischbrei schon trocken. 
Hält man den getrockneten noch nicht verpulverten Fleischbrei in einer luftleeren Glocke oder in einer Flasche unter Aetherschicht, so ist man erstaunt, wie lange derselbe seine schöne normale rothe Farbe beibehält, während er, frei an der Luft stehen gelassen, sebr bald (in ein paar Tagen) seine frische Farbe verliert und verblasst. Pulvert man das aus dem vacuo herausgenommene roth gefärbte Fleisch (Fleischbrei), so wird es, aus bekannten physikalischen Gründen, blass, rosafarben und nach stattgehabter Entfettung und nun ermöglichter feinster Verpulverung noch bedeutend blässer. Es ist daher vom verpulverten, bei höherer Temperatur getrocknetem Fleische, welches orange resp. bräunliche Färbung besitzt, schon auf den ersten Blick zu unterscheiden.

Zur Untersuchung wurde von fünf versehiedenen Ochsen (an verschiedenen Tagen) je ein grösseres Stïck aus psoas major (filet) genommen, darauf eins von den vorderen Halsmuskeln und zuletzt einem fünften Thier absichtlich ein recht sehniges Fleischstïck aus der Riickenmuskulatur (nahe der Wirbelsäule) herausgeschnitten.

Die Thiere waren mittelmässig genährt. Da es mir gar nicht darauf ankam zu erfahren, wie viel Fett im untersuchten Fleische vorhanden war, sondern es sich nur darum handelte, alles Fett daraus zu entfernen, so habe ich stets auf die sorgfältigste Entfettung geachtet, die Menge des extrahirten Fettes dagegen gewöhnlich nicht bestimmt. Nur möchte ich hier beiläutig erwähnen, dass ich mich vielfach bei Fleischuntersuchungen habe davon überzeugen können, dass auch in den Fällen, in denen am Fleische makroscopisch gar keine Fettbeimengungen sichtbar waren, dasselbe zuweilen noch beträchtliche Mengen Fett enthielt. Um ein Beispiel zu geben, enthielt der Brustmuskel einer gut genährten Taube, der makroscopisch vollkommen homogen und fettfrei aussah, noch 3\% Fett, auf feuchtes Fleisch bezogen.

Bei der Untersuchung der soeben erwähnten fünf Fleischportionen (Fleischstiicke) hat sich eine Thatsache herausgestellt, die ich näher besprechen muss. Ich batte schon früher - im Winter und im Frühjahr - mehrmals verschiedene Fleischproben aus dem Bonner Schlachthause genommen. Obwohl ich damals das Fleisch nicht sogleich in Arbeit nahm (im Gegentheil dasselbe 
öfters eine Zeitlang bei Zimmertemperatur stehen liess) und noch fencht der Einwirkung einer höheren Temperatur aussetzte, so hatten sich doch alle jene Fleischproben als ziemlich glycogenhaltig erwiesen und es enthielt jenes Fleisch getrocknet bis 2,5\%, frisch bis $0,6 \%$ Glycogen. Indessen zeigte sich merkwürdiger Weise bei der Untersuchung der so eben erwähnten fünf Fleischarten, die zu nachfolgenden Analysen dienten und die alle von Thieren stammten, die im Herbst (im September) geschlachtet waren, ein auffallender Mangel, öfters ein vollständiges Feblen von Glycogen, obgleich das Fleisch sehr schnell und nur bei Zimmertemperatur getrocknet wurde und auch nachträglich gar nicht erhitzt war.

Leider bin ich ganz ausser Stande, iiber die Ursache dieser auffallenden Thatsache berichten zu können ${ }^{1}$ ).

2. Die Analysen und deren Ergebnisse.

Wenn wir nun bei der Betrachtung der Ergebnisse unserer Analysen erst bei den Kohlenstoff- und Wasserstoff werthen bleiben, so finden wir, dass diese Zahlen für alle Muskeln auffallend unter einander tibereinstimmen und zwar in solchem Grade, wie es für eine reine chemische Verbindung nicht vollkommener hätte erwartet werden können, da die Unterschiede nicht grösser, als die Fehlergrenzen der Nethode selbst sind.

Zwar differiren die Stickst of fzahlen der untersuchten Muskeln mehr, als es in den Fehlerquellen der sehr scharfen $\mathrm{Kj}$ elda hl-W il farth'schen Methode liegt, aber die Uebereinstimmung ist noch immer eine ganz bedeutende und zwar sind die Unterschiede unserer Analysen des bis zum constanten Gewicht getrockneten Fleisches verschiedener Thiere geringer, als

1) $\mathrm{Ob}$ zufällig die schlechte Fütterung in den letzten Tagen hier die Hauptursache bildet, oder die Wärme der Jahreszeit mit im Spiel ist, weiss ich nicht anzugeben, muss aber hier darauf hinweisen, dass ich bei einem Hunde (allerdings bei reichlicher Fleischkost) sogar unmittelbar nach einer sehr grossen Arbeitsleistung noch über $1 / 2 \%$ Glycogen in dem entfetteten getrockneten Fleische gefunden habe. 
die Unterschiede zwischen den verschiedenen Analysen eines und desselben frischen, nicht getrockneten Fleisches, wie ich es durch viele Zahlen beweisen kann.

Anch die durch die Rechnung ermittelten Restzahlen für Sa u erstoff $+S \mathrm{chw}$ efel zeigen in allen Analysen eine bedeutende Uebereinstimmung, eben wegen der Uebereinstimmung der Zahlen für die anderen Fleischbestandtbeile. Die Differenz einzelner Fleischproben liegt für Sauerstoff + Schwefel innerbalb $0,1-0,3$, indem es nur einmal die letzte Zahl erreicht.

Das Verhältniss von Kohlenstoff zu Stickstoff im Fleische, (,Fleischqu ot ient" könnte man es der Kürze wegen nennen), welches eine grosse Uebereinstimmung bei einzelnen Fleischarten zeigt, schwankt zwischen 3,23 und 3,26 und ist im Mittel gleich 3,24 , steht also nahe dem von $\mathrm{Rubne} \mathrm{r}^{1}$ ) angegebenen Werthe von 3,28 .

Aber diese annähernde Uebereinstimmung der von mir gefundenen Verhältnisszahl von Kohlenstoff zu Stickstoff im Fleische mit der Rubner'schen berubt durchaus nicht auf der. Uebereinstimmung meiner Zahlen für die elementare Zusammensetzung des Fleisches mit den $\mathrm{Rub}$ e r'schen. Im Gegentheil, es zeigen unsere Zahlen für einzelne elementare Fleischbestandtheile recht grosse Unterschiede. So ist seine Zahl für Kohlenstoff um $1 \%$ höher, für Wasserstoff ebenfalls um $0,7 \%$ höher, nur für Stickstoff ist die Differenz am geringsten und beträgt nur $0,1-0,2 \%$ mehr, als die meinige; dagegen, wie es bei den eben angefubrten Zahlen nicht wesentlich anders sein kann, ist die $R u b$ n er'sche Zahl für Sauerstoff + Schwefel um volle $2 \%$ niedriger, als die meinige; endlich ist seine Zahl für den Aschengehalt ziemlich höher, als diejenige, die sich bei mir ergeben.

Man könnte vielleicht denken, dass die Differenz zwischen meinen und den Rabner'schen Zahlen vor Allem dadurch bedingt wird, dass er das Fleisch schärfer - bei höherer Temperatur - getrocknet hat. Diese Voraussetzung wird aber ganz besonders aus dem Vergleiche der $\mathrm{Rubner}$ 'schen und meiner Wasserstoffzahl hinfällig, denn bei jedem schärferen Trocknen des Fleisches - bei etwa $100^{\circ}$ und etwas darüber - vermindert sich, 
wie ich mich aus zahlreichen Analysen ïberzengt habe, der Wasserstoffgehalt im Fleische ausnahmslos, während der Wasserstoffgebalt des Fleisches bei Rubner gerade umgekehrt bedeutend höher ist, als bei mir.

Viel näher im Allgemeinen kommen meine Zahlen denen von Stohmann und Langbein ${ }^{1}$, aber gerade das Verhältniss von Koblenstoff zu Stickstoff im Muskel zeigt bei mir eine bedeutende Abweichung von der Zahl von Stohmann und Langbein. Eine Betrachtung der betreffenden Zahlen zeigt, dass meine Kohlenstoffzahl höher und meine Stickstoffzahl niedriger ist, als die von Stohmann-Langbein; daher wird natürlich der Unterschied zwischen meinen Kohlenstoff-Stickstoffquotienten und dem Stohmann-Langbein'schen ein ganz bedeutender.

Somit haben sich aus einer Anzahl von sehr ïbereinstimmenden Analysen sowohl für das Verhältniss von Kohlenstoff zu Stickstoff im Ochsenfleische, als auch für den Gehalt des genannten Fleisches an Kohlenstoff, Stickstoff, Wasserstoff und Sauerstoff bestimmte Werthe ergeben; auch erwies es sich, dass die Muskeln verschiedener Thiere derselben Spezies eine ganz auffallende Uebereinstimmung ihrer Elementarzusammensetzung zeigen ${ }^{2}$ ).

Ob auch die Muskeln bei Thieren verschiedener*namentlich wenig verwandter Arten ebenfalls gleich zusammengesetzt sind, muss vorläufig als eine offene Frage betrachtet werden. Meine geringen Erfahrungen in dieser Hinsicht würden, was den Hund betrifft, zu Gunsten einer Differenz reden, davon aber hoffe ich ein anderes Mal zu sprechen.

Bevor ich diese Blätter schliesse, möchte ich die hier beschriebene Methode, die Muskelsubstanz möglichst ohne Aenderung ihrer Zusammensetzung zur Elementaranalyse vorzubereiten, nochmals in ibrer Bedeutung für das Studium des elementaren Aufbaues der verschiedenen Gewebe, des Protoplasmas versehiedener Organe hervorheben. Es bietet dieselbe folgende Vortheile: 1) Sie umgeht die Anwendung einer erböhten Temperatur vollständig; 2) sie ermög-

1) Journ. pract. Chemie N. F. Bd. 44 S. 364 (1891).

2) Unter etwaigem Vorbehalt der ziemlich gleichen Lebensverbältnisse der Thiere. 
licht ein sehr rasches und gleichmässiges Trocknen des Gewebes bei Zimmertemperatur; 3) sie erlaubt zugleich ein längeres Aufbewahren des getrockneten Gewebes unter möglichster Ausschliessung einer chemischen Veränderung.

Meinem hochverehrten Lehrer Herrn Geheimrath P fl it ge r, dessen Anregung diese Arbeit ihre Entstehung verdankt, sage ich meinen tief empfundenen Dank. Auch meinem verehrten Freunde Dr. Max Bleibtrea bin ich zu grossem Danke verpflichtet, ebenso meinem geehrten Collegen Dr. B. Sehöndorff. Schliesslich danke ich bestens auch meinem lieben Freunde Dr. Franz Meyer.

Bemerkung zu den Tabellen:

Auf der Tabelle 1 (Ge neraltabelle) habe ich meine sämmtlichen Analysen der vier ersten Fleischproben zusammengestellt - Filet I, II, III, die Halsmuskeln -, dagegen nicht die Analysen des stark sehnenhaltigen Stücks aus den Rückenmuskeln, dessen mittlere Zusammensetzung ich gesondert fuir sich auf Tabelle 5 angeführt habe. Diese fünfte stark sehnenhaltige Fleischportion wurde absichtlich dazu genommen um ein Urtheil zu gewinnen, ob die stärkere Beimengung von Bindegewebe die Zusammensetzung des Muskels besonders alterirt. Das scheint wenig der Fall zu sein; aber nichts destoweniger habe ich bei der Aufstellung der Mittelzablen fü das Ochsenfleisch (Columne $f$ anf der Generaltabelle) nur die vier ersten Fleischarten berücksichtigt, dagegen auf der Tabelle 5, Columne b auch die Mittelzahlen für alle fünf untersuchten Fleischarten angegeben. Dieselben weichen wenig ab von den eigentlichen Mittelzahlen auf Tab. 1 Columne f. 


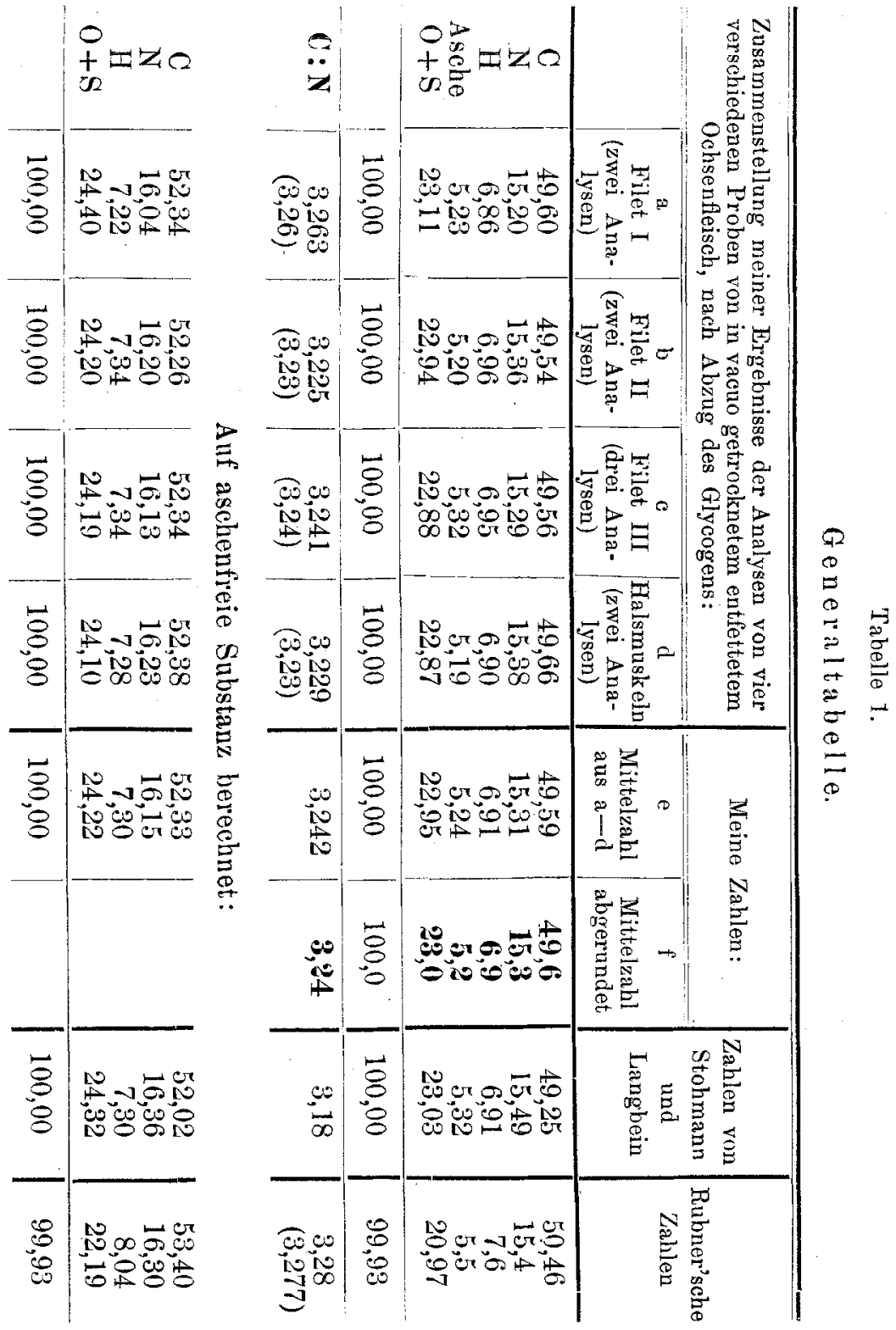


Ueber die elementare Zusammensetzung des Ochsenfleisches.

Tabelle 2.

Bestimmungen

des Kohlenstoffs und Wasserstoffs im in vacuo getrockneten entfetteten Fleische.

\begin{tabular}{|c|c|c|c|c|c|c|c|c|c|}
\hline & \multirow{2}{*}{ 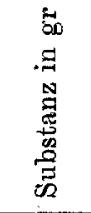 } & \multicolumn{6}{|c|}{ Erhalten } & \multirow{2}{*}{ 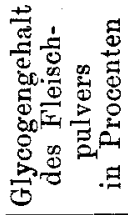 } & \multirow{2}{*}{ 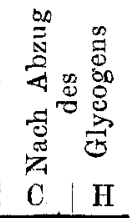 } \\
\hline & & 坣总 & 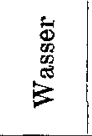 & $\mathrm{C}$ & $\mathrm{H}$ & 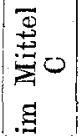 & 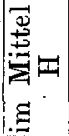 & & \\
\hline Filet I & $\begin{array}{l}0,3409 \\
0,2943\end{array}$ & 0,6209 & $\begin{array}{l}0,2092 \\
0,1824\end{array}$ & $\begin{array}{l}49,67 \\
49,53\end{array}$ & $\begin{array}{l}6,82 \\
6,89\end{array}$ & 49,60 & 6,86 & (Spuren) & $49,606,86$ \\
\hline Filet II & $\begin{array}{l}0,3460 \\
0,2845\end{array}$ & $\begin{array}{l}0,6275 \\
0,5174\end{array}$ & $\begin{array}{l}0,2158 \\
0,1787\end{array}$ & $\begin{array}{l}49,46 \\
49,61\end{array}$ & $\begin{array}{l}6,93 \\
6,98\end{array}$ & 49,54 & 6,96 & (fehlt) & $49,546,96$ \\
\hline Filet III & $\begin{array}{l}0,3881 \\
0,4826 \\
0,3497\end{array}$ & $\mid \begin{array}{l}0,7146 \\
0,8781 \\
0,6352\end{array}$ & $\begin{array}{l}0,2443 \\
0,2996 \\
0,2190\end{array}$ & $\begin{array}{l}49,52 \\
49,62 \\
49,57\end{array}$ & $\begin{array}{l}6,99 \\
6,90 \\
6,96\end{array}$ & 49,56 & 6,95 & (Spuren) & $49,0666,95$ \\
\hline & $\begin{array}{l}0,3621 \\
0,3408\end{array}$ & $\begin{array}{l}0,6569 \\
0,6239\end{array}$ & $\begin{array}{l}0,2238 \\
0,2128\end{array}$ & $\begin{array}{l}49,48 \\
49,72\end{array}$ & $\mid \begin{array}{l}6,87 \\
6,91\end{array}$ & 49,60 & 6,89 & $\begin{array}{c}1,1074 \\
(1,11)\end{array}$ & $49,66 \mid 4,95$ \\
\hline & $\begin{array}{l}0,4478 \\
0,3859\end{array}$ & $\begin{array}{l}0,8102 \\
0,6992\end{array}$ & $\begin{array}{l}0,2779 \\
0,2440\end{array}$ & 49,34 & $\begin{array}{l}6,89 \\
7,02 \\
\end{array}$ & 49,38 & 6,96 & 0,32 & $49,406,96$ \\
\hline
\end{tabular}

Tabelle 3.

Bestimmungen des Stickstoffs im in racuo getrockneten entfetteten Fleische.

\begin{tabular}{|c|c|c|c|c|c|c|}
\hline & $\begin{array}{l}\text { Substanz } \\
\text { in } \mathrm{gr}\end{array}$ & $\begin{array}{c}c c \\
\text { Titrir- } \\
\text { lauge }{ }^{1} \text { ) }\end{array}$ & $\stackrel{\mathrm{N}}{\mathrm{in} \%}$ & 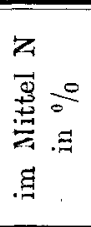 & 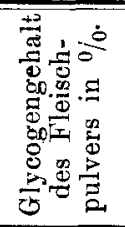 & 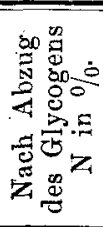 \\
\hline Filet I & $\begin{array}{l}0,4932 \\
0,4350\end{array}$ & $\begin{array}{l}=37,10 \\
=33,05\end{array}$ & $\begin{array}{l}15,13 \\
15,27\end{array}$ & 15,20 & (Spuren) & 15,20 \\
\hline Filet II & $\begin{array}{l}0,3516 \\
0,2944 \\
0,4215 \\
0,3850\end{array}$ & $\begin{array}{l}=26,80 \\
=22,40 \\
=32,25 \\
=29,50\end{array}$ & $\begin{array}{l}15,33 \\
15,30 \\
15,39 \\
15,41\end{array}$ & 15,36 & (feblt) & 15,36 \\
\hline
\end{tabular}

1) $1 \mathrm{ccm}$ Lauge $=2,01142 \mathrm{mgr}$ Stickstoff. 


\begin{tabular}{|c|c|c|c|c|c|c|}
\hline & $\begin{array}{l}\text { Substanz } \\
\text { in } g r\end{array}$ & $\begin{array}{c}\text { cc } \\
\text { Titrir- } \\
\text { lauge } 1 \text { ) }\end{array}$ & $\begin{array}{l}\mathrm{N} \\
\text { in } \%\end{array}$ & 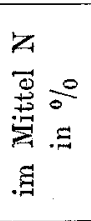 & 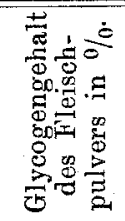 & 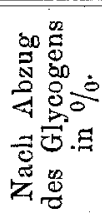 \\
\hline Filet III & $\begin{array}{l}0,7361 \\
0,7376 \\
0,7657\end{array}$ & $\begin{array}{l}=56,10 \\
=55,85 \\
=58,30\end{array}$ & $\begin{array}{l}15,33 \\
15,22 \\
15,32\end{array}$ & 15,29 & (Spuren) & 15,29 \\
\hline Hals muskeln & $\begin{array}{l}0,6517 \\
0,5622 \\
0,6371 \\
0,5377\end{array}$ & $\begin{array}{l}=49,25 \\
=42,60 \\
=48,30 \\
=40,45\end{array}$ & $\begin{array}{l}15,20 \\
15,24 \\
15,25 \\
15,13\end{array}$ & 15,21 & $\begin{array}{c}1,1074 \\
(1,11)\end{array}$ & 15,38 \\
\hline $\begin{array}{l}\text { Starksehnige } \\
\text { Rückenmusk. }\end{array}$ & $\begin{array}{l}0,7060 \\
0,5123 \\
0,5328\end{array}$ & $\begin{array}{l}=53,30 \\
=38,60 \\
=40,10\end{array}$ & $\begin{array}{l}15,19 \\
15,16 \\
15,14\end{array}$ & 15,16 & 0,32 & 15,21 \\
\hline
\end{tabular}

Tabelle 4.

Aschenbestimmungen im in vacuogetrockneten entfetteten Fleische.

\begin{tabular}{|c|c|c|c|c|c|c|}
\hline & \multirow[b]{2}{*}{$\begin{array}{l}\text { Substanz } \\
\text { in gr }\end{array}$} & \multicolumn{3}{|c|}{ Erhalten } & \multirow{2}{*}{ 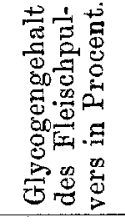 } & \multirow{2}{*}{ 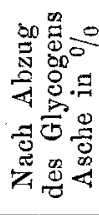 } \\
\hline & & 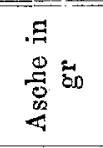 & $\begin{array}{l}.7 \\
0 \\
0 \\
0 \\
0 \\
4\end{array}$ & 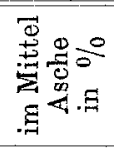 & & \\
\hline Filet I & $\begin{array}{l}1,0283 \\
1,1124\end{array}$ & $\begin{array}{l}0,0542 \\
0,0577\end{array}$ & $\left.\begin{array}{l}5,27 \\
5,19\end{array}\right\}$ & 5,23 & (Spuren) & 5,23 \\
\hline Filet II & 1,1718 & 0,0609 & 5,20 & & (fehlt) & 5,20 \\
\hline Filet III & 1,9712 & 0,1049 & 5,32 & & (Spuren) & 5,32 \\
\hline Halsmuskeln & 1,3169 & 0,0676 & 5,13 & & $\begin{array}{r}1,074 \\
(1,11)\end{array}$ & 5,19 \\
\hline $\begin{array}{l}\text { Starksehnige } \\
\text { Rückenmusk. }\end{array}$ & 2,2225 & 0,1446 & 5,15 & & 0,32 & 5,17 \\
\hline
\end{tabular}

1) $1 \mathrm{ccm}$ Lauge $=2,01142 \mathrm{mgr}$ Stickstoff. 
Ueber die elementare Zusammensetzung des Ochsenfleisches.

Tabelle 5.

\begin{tabular}{l|c|c}
\hline \hline & $\begin{array}{c}\text { Mittelzahlen für zwei } \\
\text { Analysen der starksehnigen } \\
\text { Rückenmuskeln } \\
\text { nach A bzug des Glycogens }\end{array}$ & $\begin{array}{c}\text { Mittelzahl von meinen } \\
\text { Analysen aller fünf Fleisch- } \\
\text { arten } \\
\text { (g 1 y c o g e n f r e i) }\end{array}$ \\
\hline $\mathrm{C}$ & 49,40 & 49,55 \\
$\mathrm{~N}$ & 15,21 & 15,29 \\
$\mathrm{H}$ & 6,96 & 6,95 \\
Asche & 5,17 & 5,24 \\
$\mathrm{O}+\mathrm{S}$ & 23,26 & 23,07 \\
\hline & 100,00 & 100,00 \\
$\mathrm{C}: \mathrm{N}$ & $3,249(3,25)$ & 3,24
\end{tabular}

Auf aschenfreie Substanz berechnet

\begin{tabular}{l|r|r}
$\mathrm{C}$ & 52,09 & 52,28 \\
$\mathrm{~N}$ & 16,04 & 16,12 \\
$\mathrm{H}$ & 7,34 & 7,30 \\
$\mathrm{O}+\mathrm{S}$ & 24,53 & 24,28 \\
\hline
\end{tabular}

Tabelle 6 .

Controlanalysen: Bestimmugen ron $C$ und $H$ in der Hippursäure.

\begin{tabular}{|c|c|c|c|c|c|c|}
\hline \multirow{2}{*}{ 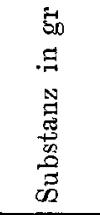 } & \multicolumn{4}{|c|}{ Erhalten } & \multirow{2}{*}{ 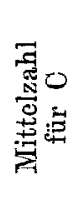 } & \multirow{2}{*}{ 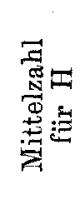 } \\
\hline & 总导 & 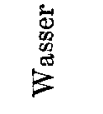 & $\begin{array}{l}0 \\
0\end{array}$ & $=\underbrace{0}_{. ;}$ & & \\
\hline $\begin{array}{l}0,4493 \\
0,3709 \\
0,4818 \\
0,3846\end{array}$ & $\begin{array}{l}0,9966 \\
0,8210 \\
1,0681 \\
0,8520\end{array}$ & $\begin{array}{l}0,2086 \\
0,1740 \\
0,2238 \\
0,1809\end{array}$ & $\begin{array}{l}60,49 \\
60,37 \\
60,46 \\
60,42\end{array}$ & $\begin{array}{l}5,11 \\
5,22 \\
5,16 \\
5,23\end{array}$ & 60,42 & 5,18 \\
\hline
\end{tabular}

Controlanalysen: Bestimmungen von $\mathrm{N}$ im Benzidin

\begin{tabular}{|c|c|c|c|}
\hline $\begin{array}{l}\text { Substanz } \\
\text { in gr }\end{array}$ & $\begin{array}{c}c c m \\
\left.\text { Titrirlauge }{ }^{1}\right)\end{array}$ & $\underset{\text { in }}{\mathrm{N}} \%$ & $\begin{array}{c}\text { Mittel- } \\
\text { zahl }\end{array}$ \\
\hline $\begin{array}{l}0,3252 \\
0,3490 \\
0,3042 \\
0,3324\end{array}$ & $\begin{array}{l}=24,5 \\
=26,25 \\
=23,1 \\
=25,2\end{array}$ & $\begin{array}{l}15,15 \\
15,13 \\
15,27 \\
15,25\end{array}$ & 15,20 \\
\hline
\end{tabular}

1) $1 \mathrm{ccm}$ Titrirlauge $=2,01142 \mathrm{mgr}$ Stickstoff. 